\title{
Contributions on Computational Intelligence in the Medical Sector
}

\author{
Helen Tucci Wang \\ Biomedical Engineering, School Engineering and Science, Stevens Institute of Technology, NJ 07030, United States. \\ wangtucci@hotmail.com; tucciwang@stevens.edu
}

\begin{abstract}
Article Info
Journal of Biomedical and Sustainable Healthcare Applications (http://anapub.co.ke/journals/jbsha/jbsha.html) Doi: https://doi.org/10.53759/0088/JBSHA202101001

Received 15 August 2020; Revised form 18 September 2020; Accepted 22 October 2020.

Available online 05 January 2021.

(C)2021 Published by AnaPub Publications.

Abstract - Patients' treatments are becoming more personalized as healthcare becomes more commodified. Meeting this need requires not just a large allocation of capital, but also a comprehensive application of information, resulting in efforts like electronic health record standards. The quantity of medical data accessible for analytics and data extraction will grow rapidly as these become more mainstream. This is accompanied by an increase in new methods for non-invasive assessment and collection of medically important data in different forms, such as signals and pictures. Despite problems with standardization and availability, the enormous quantity of data that results is a significant tool for the machine learning industry. Biomedical CI technologies are already flourishing because of getting into this data stream. The legislative session "Computer science and information Intelligence in Biology and medicine" at ESANN addresses some of the field's most pressing issues. This paper introduces the session by highlighting a few of the submissions and pointing out possibilities and difficulties for $\mathrm{CI}$ in biomedicine.
\end{abstract}

Keywords - Computational Intelligence (CI), Machine Learning (ML), Big Data (BD).

\section{INTRODUCTION}

With the current development and creation of computerized image processing, there is a desire to maximize our essential ecological assets utilizing technology, economics, and computer engineering techniques. Smart objects are technologically sophisticated machines that control how machines interact with and react to their surroundings. These technologies use computer vision to comprehend their surroundings. Advanced technologies pay attention to how users respond as their environment shifts from one media to another. Our systems are more dependable, potent, and computer technology efficient thanks to the advancements in automation systems, visual check and monitoring systems, medical and biological assessment, graphics medical diagnostic procedures, decision-econometrics smart technologies, data fusion and visual analytics, and meta-agent oriented methodologies. Biomedical computing and machine vision methods have been used in different inter-multidisciplinary fields in hybrid versions in recent years.

The aim of the proposed work is to describe current developments, implementations, and advantages in the analytical areas of data science in light of the growing problems and possibilities. Scholars from all around the globe will be able to exchange knowledge on the newly developing areas of expert machines and therapeutic image analysis as a result of this. Machine learning is propelling world-changing new technologies with the development of artificial robots. It changed the technological paradigm away from object identification and toward augmented reality and self-driving vehicles. Machine Vision is associated with picture sequences' automated extraction, attribute analysis, and usable comprehension. With the aid of vision, automatic visual comprehension and secure monitoring may be accomplished. Rehabilitation technology is also expected to be reshaped as a result of recent breakthroughs in computer engineering and cognitive technologies. These methods also aid in the detection of abnormalities in diagnostic imaging, which aid in the treatment of patients suffering from several of ailments [1]. Machine Vision methods may be the next step - and a great improvement for future healthcare bio-engineering technologies.

When creating forensic pathologist models in advanced medical sectors to assist investigative decision-making inside the 1980s, AI in healthcare was the primary focus. The utilization of statistical software, object recognition, computer vision, data structures, and dashboards for the assessment of the information and the exploration of pathways that generated the information is characterized as the recent progress of advanced computational technologies in the field of medical application. This special section provides an overview of recent developments and advantages of intelligent computing methods for face recognition systems and medical technologies, with the goal of closing the gap. The primary goal of this special section is to explain various intelligent system methods from a practical standpoint: addressing everyday issues. The main goal of this special edition is to compile up-to-date submissions on the most recent Research \& Development (R\&D) activities, as well as current problems and issues in the area of Integrated Computer Systems in Medical Computer Vision. Prospective contributions ought to be unique, unclassified, and offer new in-depth basic research discoveries from either a methodology or applications standpoint. 
The roots of contemporary science may be traced back to the Theorist René Descartes and his seminal Discourse on Methodology. When it comes to notions like projective fields, dimensions, topologies, and such, those of us working in the area of Computational Intelligence (CI) still live in his light. All of them are based on Descartes' foundational work in mathematics, and Geometric parameters are still profoundly entrenched in our scientific thinking at the most fundamental levels. It may be surprising, however, that one of mathematician's main priorities was in the pursuit of clinical information and human wellbeing. Hendrik de Roy, a medical practitioner, was one of the first opponents of Descartes' philosophy. He arguably kindled the huge discussion of the 19th Century, which built the underpinnings of medicine and technology as we understand it today, by delivering a notable and contentious lecture series on the disciplines of brain structure and the science of wellness at the University of Utretch in the Netherlands [2]. To discover a mathematical formalism of the idea of Evidence-Based Medicines (EBMs), we must go back 3 centuries. It has just been forty years since Scotland Prof. Archie Cochrane established the basis of EBM. EBM is "medical practice that focuses on incorporating knowledge gathered from latest available technologies, clinical competence, and clients' preferences and values". Its use, while controversial in some circles, is so pervasive and important to medical technology that the reputable British Medical Journal (BMJ) has named it the eighth most significant health advancement since 1840, just below the oral contraceptives and infront of computed tomography, which incorporates X-rays [3].

Health management must be based on empirical results with well-established generality, instead of on preconceptions or personal judgement, according to EBM. There's nothing new here. Admittedly, whereas EBM could perhaps regard the utilization of statistics in conjunction with other obtainable knowledge sources so in a rational style that meets the varied clinical frame of reference also has been alleged of being brainless and computational, implying a certain vilification. This interpretation may stem from the fact that, as a result of the rapid advancement of data collection and computing methodologies, the medical field is confronted with conflicting information and a great deal of data management-related strain, which can shift attention away from the patient or towards healthcare indicators and statistical evidence. While this may be a source of concern, it could also be viewed as an open ground of possibility for data analysis and the CI methodologies that are commonly associated with it. Technological developments are so important in the present medical science and healthcare delivery that they were ranked 9th in the identical BMJ poll, behind EBM. Processing of data, ironically, is poised to reverse the impression of marginalization in health insurance. Pharmacoge-nomics is a good example of this. Significant advancement in molecular genetics, as well as in technological tools such as wireless computation, cellular telephones, the internet, and computational advances in CI, have resulted in the 4P initiative of prevention measure, prescriptive, personalized, and participatory health coverage [4]. This planning is powered by not only the commercialization of health insurance enabled by new technologies, but also by a more potent factor: the fact that health insurance can only be sustained in the long run if costs per person are significantly reduced. And that implies taking care of people even when they are fit and active, with a focus on preventative medicine, keeping the people out of health facilities, prioritizing home care, and checking up on chronic illness with hospital outpatient measurement techniques that must be handled domestically utilizing automated system computation characterized by CI techniques for both personal convenience and ethics reasons.

Advisories and recommendations are then engendered and transferred in a highly efficient way, albeit with the possibility to save lives, and play an important role in slowing the current trend of rapidly rising costs. Within the next 2 decades, the 4P strategy will still have considerable effect on regular healthcare procedures. As we move forward into the twenty-first century, we will see a significant shift in the need for and application of evidence-based medicine in the provision of healthcare, with interconnected medical centers generating data more quickly than we could ever transform it over for patient care, as well as the arrival of complete genomic decryption in minutes instead of days unleashing a constant barrage of statistics at a far more quicker rate than we could ever obtain valuable information from it. The confluence of EBM and bandwidth contexts provides fertile ground for CI-based knowledge management to grow. We thought it would be appropriate to hold an ESANN panel discussion on CI in biology and medicine in this context [5]. In this article, we examine the ground of possibilities that CI methodologies open for their implementation to a field which is likely to claim them as instruments for healthcare extraction of knowledge in the future. In reality, this application field has grown so large that a guide like this one cannot possibly cover all of its key aspects. As a result; we will concentrate on the contributions by the publications acknowledged for presentation at the eighteenth International Conference held on Machine Learning (ML) techniques. In that case, this paper has been organized as follows: Section II focuses on background review of Big Data (BD). Section III

\section{BACKGROUND ANALYSIS OF BIG DATA}

\section{Scope and Definition of Big Data}

Big data is a revolutionary concept and environment in bioinformatics that allows case-based investigations to be transformed into huge, data-driven studies. The three main aspects of big data, often referred to as the 3Vs: velocity, variety, volume, are generally recognized as defining qualities of big data. First and foremost, in the biomedical computing areas, the amount of data is increasing rapidly.

ProteomicsDB8, for instance, includes 92 percent of identified genetic mutations identified inside the Swiss-Prot dataset $(18,097$ of 19,629). 5.17 TB of data is stored in ProteomicsDB. From 2009 to 2012, the advocacy of the HITECH Act 9 almost quadrupled hospital use of electronic health records (EHRs) to 44 percent. Thousands of clients' information has been gathered and kept in a digital form, with the potential to improve medical care and expand research possibilities. ten and eleven Radiology (e.g., MRI, CT scans) also generates massive quantities of data with much more complicated 
characteristics and dimensionality. The Transparent Human Initiative, for example, contains 39 GB of feminine datasets in its archives. These and other databases will enable large-scale aggregate data gathering and evaluation in the future. The diversity of data kinds and architectures is the second characteristic of big data. Many various layers of data sources make up the biological big data ecosystem, which provides investigators with a diverse set of information. Sequencing techniques, for instance, generate "omics" information almost at all scales ranging elements, from genomes to proteome to metagenomics to binding proteins to phenomics. Unstructured data (e.g., annotations in EHRs, clinical trial findings, medical pictures, and healthcare sensors) presents numerous possibilities as well as a distinct difficulty when it comes to developing novel studies [6]. The third feature of large datasets is velocity, which refers to the speed with which data is created and processed. The latest generation of sequenced technology allows for the low-cost creation of millions of Nucleotide sequences per day. Because genetic sequencing necessitates higher speeds,1,20 data mining methods will be adapted to meet the speed with which data is produced as well as the speed with which it is processed. Big data technology will also offer biological investigators with time-saving methods for identifying new trends across demographic groups utilizing data from social media in the area of public health.

\section{Big Data Tools}

Biomedical researchers are confronted with significant difficulties when it comes to storing, organizing, and interpreting large information. Big data's features need the employment of advanced and innovative techniques to extract valuable information and allow more widespread healthcare solutions. We observed that several technologies, including as artificial intelligence (AI), $\operatorname{Hdfs}{ }^{\circledR}$, and machine learning tools, were utilized jointly in the majority of the instances reported. One of the most major infrastructure projects for tackling large data problems is parallel computation. On a network of devices or quantum computers, it may do algorithm jobs at the same time. New parallel computing approaches for a new large data infrastructure, such as Google's MapReduce, have already been suggested in recent decades. Apache has just launched Hadoop, a decentralized database processing accessible MapReduce program. Concurrent network connectivity to clustered computers is supported by Hadoop Distributed File Systems (HDFSs) [7]. Hadoop-based applications may also be thought of as cloud computing platforms, since they provide centralized information storage and remote monitoring via the Web. As a result, cloud computing is an emerging paradigm for sharing customizable computing capacity over the networks and it may be used as an architecture, framework, and/or application to provide a sustainable solution. Cloud computing may also enhance system's performance, responsiveness, and adaptability by reducing a need to manage hardware and/or software capabilities and using less assets for maintenance services such as deployment, setup, and testing. Cloud services are used in many innovative big data systems.

\section{BIG DATA APPLICATIONS}

\section{Bioinformatics applications}

Bioinformatics study examines molecular changes in biological systems. With today's customized medical trends, there's a growing necessity to produce, store, and assess massive amounts of data in a more reasonable amount of timeframe. Genomic data may be obtained in a short amount of time thanks to next-generation sequencing technologies. Big data methods in bioinformatics technologies help scientists collect and analyze biological data by providing data repositories, computational infrastructure, and fast data manipulation tools. In [8], researchers explain how Mapreduce and Hadoop are being utilized in the biomedical sector right now. Big data devices and systems are divided into four areas in this section: Keeping and retrieving information; identification of errors; examining the data; and storage and retrieval of data. These areas are interlinked and can intersect; for instance, more datasets inputs can enable foundational analysis of data. Beside in this research, we solely classified technologies based on their primary purposes.

\section{Keeping and Retrieving Information}

In today's world, a single run of a sequencing machine may generate millions of short DNA sequence information. To be utilized for further research, such as genotyping and expression variation analyses, the sequencing data must be mapped to particular reference genomes. CloudBurst is a distributed processing approach for genome mapping that speeds up the procedure. To improve the sustainability of sizable sequencing data processing, CloudBurst makes parallel the short-read fourier transform. The CloudBurst concept was sorely assessed on a 25-core ensemble, and the results showed that it handled 7 million short-reads almost 30\% faster than a single-core machine [9]. New CloudBurst-oriented biomedical research technologies created by the CloudBurst team include Contrail for reconstructing large genome and Slingshot for identifying single nucleotide polymorphisms (SNPs) from genome sequencing.

DistMap is a toolset for Hadoop clusters that allows for distributed short-read mapping. DistMap seeks to broaden the support for various mappers in order to cover a broader variety of sequencing applications. TopHat, BSMAP, MISMARK, STAR, SOAP, GSNAP, BOWTIE2, BWA are among the nine mapper types that are handled. DistMap has a mapping process that is controlled by simple instructions. A 13-node cluster, for example, was used in an assessment test, proving that it is a useful technique for measuring short-read set of data. Distmap permits BWA mappers to complete half a billion read pairings (248 gigabytes) in 6 hours that is 3 times quicker compared to a solitary mapping. SeqWare is a querying processor for biomedical scientists that are built on top of the Hadoop HBase database. It allows them to access huge whole-genome datasets. To connect genome browsers and tools, the SeqWare team developed an interactive interface. As portion of a prototyping investigation, the 1102 GBM \& U87 MG tumour datasets were exported, and the scientists used these engines to assess the Berkley DB and Hdfs rear fenders for exporting and importing variable capabilities [10]. The 
results show that the Berkley DB approach is quicker whenever scanning $6 \mathrm{M}$ variances, whereas the HBase approach is superior when scanning more than 6 million iterations.

The Japanese DNA Data Bank (DDB) has developed cloud-oriented workflows for throughput assessment of the data sequences for upcoming generations known as the Read Annotation Pipeline ${ }$. This cloud-computing system was created by DDBJ to help in sequence analysis. It has a simple user interface for processing sequencer data, with two levels: (1) the entry-level framework considers the FastQ metadata and pre - process it to level of external bases. The second analysis involves mapping the data to genomic references or putting it together on supercomputers. SNP identification, RNAsequencing (RNA-seq) assessment, and ChIP-seq assessment are all performed using the Galaxy interface in this process. DDBJ mapped 34 million sequenced communicates to the $383 \mathrm{MB}$ genome sequence in six hours during benchmark testing. Hydra is a Hadoop-based, scalable proteome search engine. Hydra is a computer program for processing huge peptides and spectroscopy databases that uses a parallel processing architecture to enable scalable spectrometer data retrieval. Hydra divides the proteome search into two parts: (1) creating a peptide repository, and (2) rating spectrum and obtaining data. On a 43-node Apache hadoop, the program can score 27.1 billion peptides in less than 1 hour.

\section{Identification of Errors}

There have been a variety of methods created to detect mistakes in sequencing data; SAMQA detects such problems and guarantees that huge genomic data satisfies minimal quality requirements. SAMQA includes a set of technological checks for detecting data abnormalities such as blank runs (e.g., homology modeling mapping formatting issue, wrong CIGAR values). It was initially designed for the Department of Medicine Genotyping Array to identify and disclose errors rapidly. For biological evaluation, scientists may devise a criteria to screen out possibly incorrect results (empty reads) and submit them to experts for manual evaluation. When contrasting Hadoop, which has been examined on a system, to SAMQA, that was examined on a single machine, the Distributed system processed a 23 GB sample approximately 80 times faster (18 hour shifts) [11]. For three main sequencing portals: Reading, Helix, and SOLiD, ART offers simulation results for sequencing analysis. ART has read error or reading length profiles built in, and it can detect three kinds of sequencer inconsistencies: base replacements, penetrations, and removals.

CloudRS represents a higher-throughput sequencing information and data error-rectification technique oriented on a scalable and parallel architecture. The RS algorithm was used to create this technique. The CloudRS researchers used the GAGE benchmarks to test the system on six distinct datasets, and the findings indicate that CloudRS has a better accuracy rate than the Reptile approach.

The Genome Analysis Toolkit (GATK) is an Apache hadoop programming framework intended to enable large-scale Genomic DNA processing, in addition to the mentioned framework and development tools for sequencing data analysis. GATK supports a variety of data formats, including SAM recordings, bilateral alignments maps (BAM), dbSNP, and HapMap, GATK's "Traverse" components compile and load sequencing packets into the system, offering relevant links to the databases, like database categorization by locus.

The "walkers" component utilized datasets and generates analytics results. The Genome Sequencing Atlas and the 1000 Genomes Projects both utilized GATK. The ArrayExpress Library of Functionality Sequencing data source is a global effort to bring high-throughput genome dataset inclusively. The collection contains thirty thousand experiments more than 1 million trials. Approximately 80 percent of the data was retrieved from the GEO data provider, with the remaining 20 percent supplied immediately to Array Express with its customers. On a daily basis, and over 1,000 unique users use the platform, downloading more than $50 \mathrm{~GB}$ of data. For data transfer and analysis, the platform additionally integrates with $\mathrm{R}$ and GenomeSpace.

\section{Storage and Retrieval of Data}

It is important to talk about how big data methods (like Hadoop and NoSQL databases) are utilized to store EHRs. When dealing with therapeutic real-time stream data, effective data storage is particularly essential. Many studies evaluated Hdfs and Apache hbase as file storage for gathering EEG data and emphasized their elevated characteristics. Researchers explored the potential of Apache and Hdfs for decentralised EHRs. Many academics have also proposed a decentralized option for managing and retrieving massive amounts of EEG output. Cloudwave, their system, stores clinical data using Hadoop-based data processing modules, and they created a web-based frontend for actual data visualisation and retrieving using Hadoop's processing capability. The Cloudwave team tested Cloudwave against a stand-alone system using a database of 77 GB EEG signal information; the findings indicate Cloudwave tested 5 different EEG trials in a single minute, whereby the single-based program tests for more than 20 minutes. Whenever contrasted with the more conventional dataset systems that succeed when it comes to dealing with structured datasets, NoSQL is considered an excellent option for the storage of more complex datasets. Analysts developed a system that enables data mining methods while also providing agility and responsiveness in data processing by combining database and multivariate technologies with NoSQL repositories. The statistics of clinical instruments is saved inside HBase in such a manner that the row key acts as the time stamp for the arithmetical findings and the column integrates the patient's physiological values, which are similar to the row major timestamp. The information for the HBase data structure is kept in MongoDB, a document-based NoSQL database, to enhance accessibility and readability. The Search Engine Toolkit is used to display the clinical signaling data in the system.

\section{Data Retrieval for Data Sharing}


In order to share medical knowledge and integrate data, interactive health information extraction is anticipated to play a significant role. Many scholars have recognized the necessity for such a position and proposed solutions. To address the limitations of cloud-enabled online communities for eHealth solutions, they suggested a three-tier ecosystem. The scientists created a method for interoperable EHRs that are cloud-based. Sharp suggested cloud-based application architecture to improve collaboration amongst investigators in multi-tenant clinical studies. They used a cloud-based method to explore the current and future features of translational informatics. They developed private public cloud architecture for dealing with large data demands from healthcare practitioners. They designed the "Healthcare Data Handling and Analyzing System" in China to handle massive quantities of online cardiovascular disease data analysis. They utilized a hybrid XML databases and the Hadoop HBase architecture.

\section{Data Protection}

Data analysts found that large datasets can be gathered over time, and that big data possibilities may be used to meet and solve health-care problems. As a result of these advances in data technology, health-care practitioners will be able to manipulate each quantity of datasets in the upcoming generation. More interactive retrieval of data, on the contrary, puts a higher strain on data security. MedCloud is a concept suggested that uses the Hadoop environment to address HIPAA compliance concerns while accessing the datasets for patients. Home-Diagnosis, a cloud-based platform for addressing privacy issues has been developed, ensuring highly concurrent and scalability patient records retrieving, and conducting data analysis in a self-care environment. In Home-Diagnosis, a Lucene-oriented disseminated search clustered was used to focus on the major issues whereas Hadoop clusters were utilized to increase the speed on the entire process.

Developing simulation techniques for therapeutic prognosis is a complex process, and forecasting illness risks and progression over times may be very useful for clinical information systems. PARAMO is introduced as a computational modeling system for analyzing computerized patient data. PARAMO enables you to build and use a medical information analytic pathway for a variety of complex models. PARAMO successfully executes concurrent tasks by using Hadoop that gathers data for a massive amount of medical data that can be processed in a fair amount of time. The PARAMO platform was connected with healthcare language taxonomies (e.g., UMLS, ICD etc.), and the study was applied on a sample of Electronic health records spanning from 4, 000 to 299, 000 participants that used a Distributed architecture having 10 to 150 concurrent jobs. On this big dataset, the results demonstrate that running 160 concurrent processes is 72 times quicker than running 10 concurrent jobs [12].

Furthermore, massive data techniques are used to assess the monthly risks of admission in patients with congestive heart failure. The National Health and morbidity Dataset and the Medical centre Healthcare System were used to retrieve patient information. To assess the probability of patient readmission, many methods (e.g., regression, randomized forest) were employed to construct a prediction model. The researchers ran a number of tests on over three million patient data. The findings indicated that using big data improved the performance of constructing a forecasting model substantially: the models had the greatest accuracy $(76 \%)$ and recollection $(62 \%)$. Data analysts presented MapReduce-oriented data-based prototypes for treating Hypertrophic Cardiomyopathy (HCM), a genetic heart condition that causes cardiac mortality in young athletes. Due to the vast number of possible factors, a successful identification of HCM is difficult. A data-driven analysis might increase the diagnostic rate [13]. In addition to increased prediction accuracy, the testing findings revealed that while retrieving a dataset of 9000 actual medical records, the total runtime of the forecast modeling dropped from 9 hours to a few minute. This is a significant advancement over prior studies, and it may pave the way for future uses in early systematic diagnosis.

Additionally, using big data to evaluate clinical data has the potential to have a major effect on the medical world. Future applications of advanced analytics have been described by many academics. The adoption of picture archiving and communication systems (PACSs) and electronic health records (EHRs) has led in the accumulation of massive quantities of digital massive data. They also found that urologists might use predictive analytics to help them make decisions, such as predicting if patients might require hospital readmission after a cystectomy. Data analytics may be used to decide whether a 75-year-old patient should get radiation treatment or a prostatectomy in order to prevent imminent dangers from advanced breast cancer. Analysts spoke through how big data may help with things like determining the cause of a patient's symptoms, forecasting the risk of illness development or recurrence, and enhancing primary care quality. The big data approach is a novel tool for discovering meaningful connection among huge quantities of "chaotic" medical studies. Furthermore, using a big dataset will allow gastroenterologists to quickly increase their knowledge of digestive disorders. Analysts presented a patient-centered framework while illustrating the broader goal of the big data approach to customized medicine. Big data has a role in perioperative care. Big data may assist identify fatal pediatric therapeutic complications at an initial phase, resulting in a revolution in therapeutic trials for premature infants. An active lifestyle system is suggested, claiming that a graphic aesthetic engages users by increasing self-motivation.

\section{Image Informatics Application}

The study of techniques for producing, organizing, and representing imaging data in different biological applications is known as imaging informatics. It is focused with the sharing and analysis of medical images in complicated health-care systems. The need to integrate radiology data into EHR systems is quickly raising as the need for more customized treatment grows. Imaging informatics arose nearly concurrently with the introduction of electronic health records (EHRs) and the development of clinical bioinformatics; nevertheless, it differs from health informatics owing to the diverse data types produced by various clinical imaging modalities. Dataset securities is still a fundamental concern in this segment; however, there is little study focused on enhancing data security in neuroimaging informatics since present systems mainly 
depend on commercialized cloud applications and established standards, such as computer vision communications in medicine.

\section{CI IN BIOMEDICAL SCIENCE}

Dimensionality Reduction in Biomedical Issues

Medicine has long been a specialized field for statistics. The fundamental significance of statistics in medical technology has long been recognized, and its goals include healthcare handling and observation, detection of diseases, management, and assessment, and the review of disease therapies. The intricacy of biological information necessitates going beyond statistical inference and relying on information retrieval (to perform data preparation procedures. The burden of dimensions is one of the key and most typical issues posed by biological data. These types of information are frequently not gathered with the express intention of data collected modeling, and one of the undesirable effects is the all-toocommon circumstance in which only a small number of information records with a high dimension are accessible for examination. With the introduction of high genetic and proteomics techniques, this has never been more apparent than in the omic group. For tiny data sources with high dimensions, relatively few conventional statistical models (and the same may be true for CI approaches) scale effectively.

Due to information scarcity, the incredibly high regions in which information is stored are intrinsically scant, resulting in unanticipated geometric features and issues with statistical measures. The issue of large dimensions in information is likewise one of modeling openness. However, one of the difficulties of using CI approaches in the biological field (specifically in daily clinic settings) is that the findings they provide are often restricted in their understanding. This is, without a doubt, a delicate topic in the scientific realm, and one that should not be overlooked: Absence of conversion into usable medical expertise can convey even the best efforts in state system information modeling methodologies ineffective. As noted in the preface, one option to improve models clarity is to illustrate how CI strategies work using rule methodologies. In contrast, dimensionality reduction (DR) might make it easier to understand the findings. Different parameters can be used to classify DR approaches. There are two types of DR techniques: regular and quadratic.

There are techniques of Feature Selection (FS) that intend to identify one or multiple prudent subgroups of characteristics that are ideal as per a specific factor (for classification, estimation, erroneous reductions, etc.), and strategies of Feature Extraction (FE) that merge the obtainable information in various aspects to develop additional, relatively small types of features to replace the initial ones. The various techniques have their merits and demerits that are outside the scope of this lesson. The use of FS and FE for DR is described in relation to a challenge of individual brain tumor classification. It is claimed here that, while Probabilistic Components Analyzing is a popular FE approach in this situation, it does have drawbacks, one of which being the original point. Because of this, it is worthwhile to pursue the design and application of better specialized FE approaches. The suggested Spectroscopy Pattern Retrieval method was established inside a strong nonlinear Bayesian approach that does not sacrifice comprehensibility while being tailored to spectra information's unique qualities. A unique technique to DR can be explored in (20 and 21). Here, relatively low manifold representations are used to simulate extremely high information.

The Portrayals are intended to offer a mechanism for the graphical representation of information and outcomes, as well as records on the organizational architecture of statistics. Visual is a very strong technique for gaining exploration information understanding. In [14], a Bayesian approach of the manifold training category is used to assess electromyographic (EMG) information records pertaining to stroke individuals receiving rehabilitative therapies, comparable to the one employed in [15]. This is a restricted HiddenMarkov Models that performs well in noisy environments and is well adapted to the study of multimodal timed sequences like the EMGsignal at hand. In the research, a more traditional Self-Organizing Map (SOM) approach is utilized to assess both physical data and therapy features in acute renal insufficiency individuals. In the first phase of the analysis, SOM is employed to obtain meaningful information via representation.

\section{Biomedical Pharmacy}

Since the late 1950s, medical decisions assist structures have been using CI approaches. Artificial neuron network had more than 1,000 references in scientific research by 1995. A simple research in a biomedical dataset such as PUBMED now yields over 17,000 responses. When the phrase "drugs surveillance" is included in the research, the count drops dramatically. To be more specific, a PUBMED research using the phrases "neuron network" and "drugs surveillance" yields just 18 findings, all of which are current (only 2 findings if the analysis is narrowed to the concepts "artificial neural networks" and "substance surveillance"). All of this points to the fact that the use of neuron models as an useful approach for therapeutic drug monitoring (TDM) has only lately arisen (in comparison to other biological devices), becoming a potential and emerging field from both a practical and theoretical standpoint. The importance of computer-based statistical analytics in pharmaceutics has risen dramatically as a result of the large quantities of data available about medicines and therapeutic responses. CI approaches can aid in the extraction of information from these datasets, giving doctors effective decision-making aids.

Many medications have unknown effects, particularly those with limited therapeutic limits that are highly essential to patient variables. In certain situations, any tool that may help determine the appropriate amounts to deliver is critical in order to prevent overdosing (possible overdoses) or underdoses (no effect of the medication on the condition of the patient). Significant breakthroughs in dose formulation, TDM, and the arising significance of combination therapy have lately contributed to a significant increase in patients' satisfaction with lifestyle. Nonetheless, the growing amount of information being gathered, as well as the multidimensional character of the fundamental pharmacokinetics and pharmacological mechanisms, justifies the creation of mathematical methods effective for forecasting concentration of the 
drug and subsequently modifying the ideal prescription. To estimate blood levels, physiological model of pharmaceutical intake and dispersion, Bayesian prediction, neural and kernel approaches have all been applied. There have also been very few attempts made focused on Supervised Learning; to find the best guidelines (clinical procedures for prescribed medications) to attain a specific goal (typically defined by a recipient's condition). Sun and associates offer Gaussian Processes (GPs) with various correlation measures as classifiers of per-meability coefficients in Humans, Pig, Rodent, and Stretchable membrane in our specialized conference. Since the distribution of pharmaceuticals via broken skin has become a routine in recent decades, this is not just a difficult challenge, but also a particularly important one in the context of biological pharmacology. The statistics reveal that GPs outperform QSAR classifiers, particularly when Parametric and neuron networks correlation models are utilized. Overall, while using CI approaches to Biological Pharmacology has generated some promising discoveries, there still is a long way to go in this field of study, which is projected to see a lot of activity in the coming decades.

\section{Cancer Assessment and Survival}

The initiation of the World Wide Web (WWW) judgment endorses mechanism Adjuvant piqued physicians' curiosity in WWW judgment assistance structures, particularly for breast cancer. The use of neuron internet methodologies for failed time information, also identified as sustenance designs, has a long history, dating back to the business of Adjuvant founders. Nevertheless, the intelligent decision care package is based on performance from metaphysical customized to a specific US set of data and publicly confirmed on data obtained for the British Columbia Cancer Agency in Vancouver, rather than a new data approach. The field's objective is to develop flexible methods that can fit information reliably without requiring proportional hypotheses, as well as in the existence of time apparent effects and nuanced interaction among variables. Normal medical predictive methods often depend on these hypotheses, or hand-crafted adjustable alternatives to get around them, which necessitates this necessity. The non - linearity of medical information, in specific, has resulted in the classification of critical prognosis variables, such as histologic stages, compromising their stability.

The existence of generally perfect right information, that is, knowledge concerning a generally lower on the anticipated occurrence dates, is a crucial aspect of prognosis concepts for cancer. For instance, if a victim dies from a condition unassociated to cancer, data about cancer return is unavailable beyond that period. Modeling the accumulated chances for the danger of the event occurring emerging after any specified season, similar to straight modeling preservation, as well as modeling the situation possibility of the occasion happening during a separate given period, constraint on it not having taken place prior to the start of that sequence. This is referred to as hazardous modeling, and it was first presented with the Partial Logistic Artificial Neural Network (PLANN). Rigid Bayesian frameworks used for artificial neural topologies, such as the Multi-Layer Convolution are another option [15].

These methods are a hybrid of traditional analytics and computer intelligence. To differentiate across individual cohorts at significantly different mortality risk or return, all of these approaches must stabilize flexibility with strong generalization abilities. This necessitates the use of complication control applications, such as the preceding Bayesian techniques and proof approximations with Automatic Relevance Determination (ARD). Furthermore, computational methods must be incorporated into diagnostic integrations that are understandable to physicians, and efforts have been made to segment patient populations by stake and clarify the designations using moderate Boolean regulations, both to validate the neurological platform's process against specialist medical knowledge and to clarify the predictive approach by substituting the analysis black-box architecture with a predictive regulation tree. Some of these algorithms have been enhanced to predict more than one competitive hazard, such as localized vs. remote tumor recurrent. When only time-tofirst-event is available and all danger hazards estimates must sum up to one, this can be assured, for example, by applying the well-known super soft activating value and ensuring a consistent relationship with the optimization techniques for statistical logistic regression. More recent, the use of kernel approaches to survival, such as the Support Vector Machine, has gotten a lot of interest. This is a new field that aims to take advantage of the exceptional discriminating capability of techniques from computation training concepts while applying them to failed time information with censoring. The very last contribution in this official session is a solid example of the state at the time in this field.

\section{CHALLENGES AND OPPORTUNITIES FOR CI AND BD IN BIOMEDICAL RESEARCH}

Medical science has evolved into a data-intensive field of study in the past decade, with new data collection methods appearing at an alarming rate. Improvements in disease research, among the most dynamic medical applications, were appeared on the front of one of Nature's most recent editions. Several next-generation sequencing methods were developed with the specific goal of monitoring genetic alterations in tumor cells, even within the extremely narrow framework of only this particular topic and study field. To discover single-nucleotide variations in the human genome, state-of-the-art data collection was coupled with sophisticated CI methods in the shape of Bayesian mixturemodels. The increasing use of microarrays in genetics and proteins chips and tissue panels in proteonomics has increased the amount of information on active metabolic pathways accessible from (f) MRI, PET, microscopic CT, and MR spectroscopy. The diversity of accessible medical information, which includes expert information on disease variability, particularly in the makeup of cancer samples, as well as clinical signs that are frequently systemic in character, further complicates an already multifaceted picture. These indications are often represented using discrete data, as opposed to tonumeric physiological measures. The rise of greater genomic technology is requiring a thorough re-evaluation of not just data management and processor protocols, but also of biological internet connectivity on the internet and in interactive grids. The requirement for physician assessment, patient presentation, and regulatory demands for verification and safety monitoring are all issues. They all emphasize the need of expressing the functioning of modeling techniques in simple Boolean terms, complete with 
filtering for individuals whose threat balance favors or opposes certain treatment options. In this regard, there has been a lot of interest in the application of rule derivation from CI systems in biomedical interest in recent decades, with a lot of it focused on disease data gathering.

\section{CONCLUSIONS AND FUTURE RESEARCH}

Computational intelligence in biomedical science is a rapidly growing area driven by ever-increasing volume of data. It is up to the scientific community, and the therapeutic and IT businesses, to cooperate to create new machine learning methods and verify them properly, such as by using methodologies and finding out how to convert findings into smooth interfaces for regular medical care. Computational intelligence offers a lot of potential in three biological applications: principal component analysis, functional genomics, and logistic regression. While considerable progress has been made in all of these areas, as shown by the examples presented in this session, these disciplines are still in their childhood, with far more research to be performed. While big data has tremendous potential for enhancing health care, all four industries confront comparable challenges when using the technologies; the most significant of them is data interoperability. VISTA, for instance, is a cluster of 128 linked systems instead of a unified system used by the VHA. When networks include a different types of data (for example, incorporating an image datasets or even a database of laboratory results into existing systems), this becomes considerably more complicated, limiting the capacity of a system to question the entire system to retrieve patients' records. The deficiency of consistency of lab figures and techniques makes data integration even more difficult. When image data is collected from multiple laboratories using different methods, for example, technical batching effects may occur. When a batch effect occurs, attempts are undertaken to normalization; although this is simpler with picture data, it is intrinsically more challenging with scientific test data. Privacy and security issues continue to be significant barriers to massive data integration and application in the mentioned four domains, requiring the creation of secure platforms with effective communication standardization. To mention a few potential catastrophic consequences, enhance the strength of applicability, boost the precision of risk prediction, integrate adaptability to huge datasets, and integrate various signal modalities in a single unified model, like numerical information generated from clinical signals coupled with qualitative categories indicating clinical state and cardinal parameters from populations.

\section{References}

[1]. S. Wiwanitkit and V. Wiwanitkit, "Computational intelligence in tropical medicine", Asian Pacific Journal of Tropical Biomedicine, vol. 6, no. 4, pp. 350-352, 2016. Doi : 10.1016/j.apjtb.2015.11.009.

[2]. Q. Zou, L. Chen, T. Huang, Z. Zhang and Y. Xu, "Machine learning and graph analytics in computational biomedicine", Artificial Intelligence in Medicine, vol. 83, p. 1, 2017. Doi : 10.1016/j.artmed.2017.09.003.

[3]. "Special issue on computational intelligence in medical systems", IEEE Transactions on Information Technology in Biomedicine, vol. 10, no. 4, pp. 813-813, 2006. Doi : 10.1109/titb.2006.884979.

[4]. H. Anandakumar and K. Umamaheswari, "A bio-inspired swarm intelligence technique for social aware cognitive radio handovers," Computers \& Electrical Engineering, vol. 71, pp. 925-937, Oct. 2018. doi:10.1016/j.compeleceng.2017.09.016

[5]. R. Arulmurugan and H. Anandakumar, "Early Detection of Lung Cancer Using Wavelet Feature Descriptor and Feed Forward Back Propagation Neural Networks Classifier," Lecture Notes in Computational Vision and Biomechanics, pp. 103-110, 2018. doi:10.1007/978-3319-71767-8_9

[6]. I. Bichindaritz, "Memory Organization As The Missing Link Between Case-Based Reasoning And Information Retrieval In Biomedicine", Computational Intelligence, vol. 22, no. 3-4, pp. 148-160, 2006. Doi : 10.1111/j.1467-8640.2006.00280.x.

[7]. "Call for Papers - A special issue of Computational Intelligence Magazine on Computational Intelligence", IEEE Computational Intelligence Magazine, vol. 3, no. 2, pp. 75-75, 2008. Doi : 10.1109/mci.2008.919092.

[8]. D. Lai, R. Begg and M. Palaniswami, "Computational Intelligence in Gait Research: A Perspective on Current Applications and Future Challenges", IEEE Transactions on Information Technology in Biomedicine, vol. 13, no. 5, pp. 687-702, 2009. Doi : 10.1109/titb.2009.2022913.

[9]. A. Malik., "Ten simple rules for engaging with artificial intelligence in biomedicine", PLOS Computational Biology, vol. 17, no. 2, p. e1008531, 2021. Doi : 10.1371/journal.pcbi.1008531.

[10]. H. Anandakumar and K. Umamaheswari, "A bio-inspired swarm intelligence technique for social aware cognitive radio handovers," Computers \& Electrical Engineering, vol. 71, pp. 925-937, Oct. 2018. doi:10.1016/j.compeleceng.2017.09.016

[11]. R. Arulmurugan and H. Anandakumar, "Early Detection of Lung Cancer Using Wavelet Feature Descriptor and Feed Forward Back Propagation Neural Networks Classifier," Lecture Notes in Computational Vision and Biomechanics, pp. 103-110, 2018. doi:10.1007/978-3319-71767-8_9

[12]. R. Rada, "Artificial intelligence", Artificial Intelligence, vol. 28, no. 1, pp. 119-121, 1986. Doi : 10.1016/0004-3702(86)90034-2.

[13]. R. Chrisley, "Embodied artificial intelligence", Artificial Intelligence, vol. 149, no. 1, pp. 131-150, 2003. Doi : 10.1016/s0004-3702(03)000559.

[14]. A. Haldorai and A. Ramu, "Canonical Correlation Analysis Based Hyper Basis Feedforward Neural Network Classification for Urban Sustainability," Neural Processing Letters, Aug. 2020. doi:10.1007/s11063-020-10327-3

[15]. D. Devikanniga, A. Ramu, and A. Haldorai, "Efficient Diagnosis of Liver Disease using Support Vector Machine Optimized with Crows Search Algorithm,” EAI Endorsed Transactions on Energy Web, p. 164177, Jul. 2018. doi:10.4108/eai.13-7-2018.164177 